\title{
Massive splenic infarction in a patient with pneumococcal septic shock and unknown celiac disease
}

\author{
Alessandro Graziani, ${ }^{1}$ Pierpaolo Casalini, ${ }^{2}$ Federica Mirici Cappa,${ }^{3}$ Francesco Albertini, ${ }^{4}$ Erica Fiorini, ${ }^{3}$ \\ Giampaolo Ugolini, ${ }^{5}$ Leonardo Rasciti ${ }^{1}$ \\ ${ }^{1}$ Internal Medicine Department, S. Maria delle Croci Hospital, Ravenna; ${ }^{2}$ Intensive Care Unit, Faenza Hospital, Faenza (RA); \\ ${ }^{3}$ Internal Medicine Department, Faenza Hospital, Faenza (RA); ${ }^{4}$ Infective Care Department, S. Maria delle Croci Hospital, \\ Ravenna; ${ }^{5}$ Surgical Department, Faenza Hospital, Faenza (RA), Italy
}

\begin{abstract}
Splenic infarction (SI) is a rare event occurring when the splenic artery or its branches become occluded by embolus or by in situ thrombosis. Many SI events are a result of embolic sources either cardiac or aortic. Massive splenic infarction (MSI) results from compromised blood flow to more than half of the spleen. In this paper we describe a case of a previously healthy patient who presented with pneumococcal sepsis who, upon investigation, revealed an unknown celiac disease and a MSI. Abdominal ultrasound with contrast agent was a useful tool for a diagnosis and follow up of this patient.
\end{abstract}

\section{Imaging in Internal Medicine}

Massive splenic infarction (MSI) results from compromised blood flow to more than half of the

Correspondence: Alessandro Graziani, Internal Medicine Department, S. Maria delle Croci Hospital, viale Missiroli 10, 48121 Ravenna, Italy.

E-mail: alessandro.graziani@auslromagna.it

Key words: Splenic infarction; endocarditis; pneumococcal sepsis; ultrasound contrast.

Conflict of interest: the authors declare no potential conflict of interest.

Received for publication: 14 July 2017.

Revision received: 24 August 2017.

Accepted for publication: 25 September 2017.

This work is licensed under a Creative Commons Attribution NonCommercial 4.0 License (CC BY-NC 4.0).

(C) Copyright A. Graziani et al., 2017

Licensee PAGEPress, Italy

Italian Journal of Medicine 2017; 11:407-408

doi:10.4081/itjm.2017.908 spleen. SI may develop secondary to cardiovascular disorders as atrial fibrillation, ventricular aneurism and heart valve diseases. Other etiological non-cardiovascular disorders are hematological diseases, thrombotic diseases, collagen tissue diseases, trauma, abdominal surgery or infection. The predominant symptom is abdominal pain, sometimes acute and severe, located in the left upper quadrant associated with fever and vomiting. The laboratory exams may show an increased serum lactate dehydrogenase and white blood cells. The abdominal computed tomography (CT) with contrast medium can demonstrate signs of vascular thrombosis and a loss of parenchymal vascular signal. ${ }^{1}$ We observed a 57-year-old woman admitted to our Intensive Care Unit (ICU) for pneumococcal septic shock with disseminated intravascular coagulation and purpura fulminans. ${ }^{2}$ During the hospitalization, she underwent an abdominal echo-Doppler with contrast agent that revealed a complete loss of splenic perfusion which suggested a MSI (Figure 1). This datum was confirmed subsequently also by abdominal

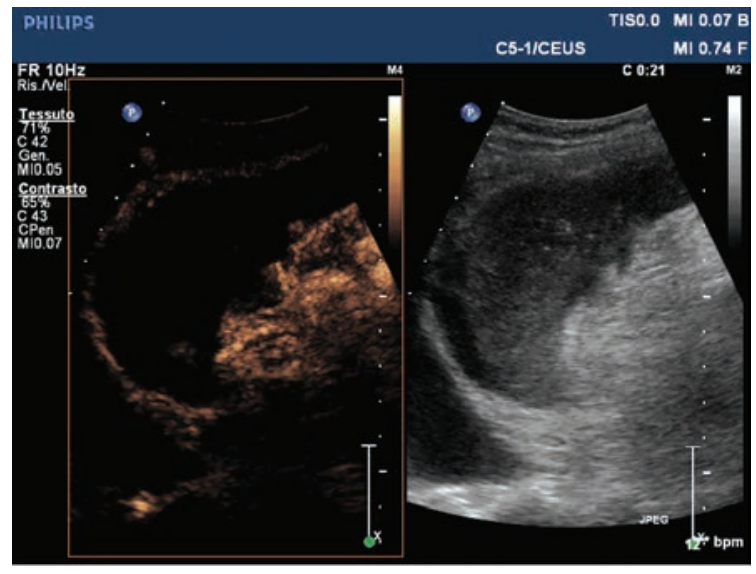

Figure 1. Abdominal echo-Doppler with contrast agent revealing a complete loss of splenic perfusion suggestive of massive splenic infarction. 
CT with contrast material (Figure 2). Transesophageal echocardiogram demonstrated a vegetation on the mitral valve, not showed by a former transthoracic examination. Laboratory investigations also showed a positive serology marker for cardiovascular disease (anti-gliadin and anti-endomysial antibodies) subsequently confirmed by an upper gastro-intestinal endoscopy. ${ }^{3}$ We would like to emphasize that in patients with a massive bacteremia caused by encapsulated microorganisms and no obvious pri-

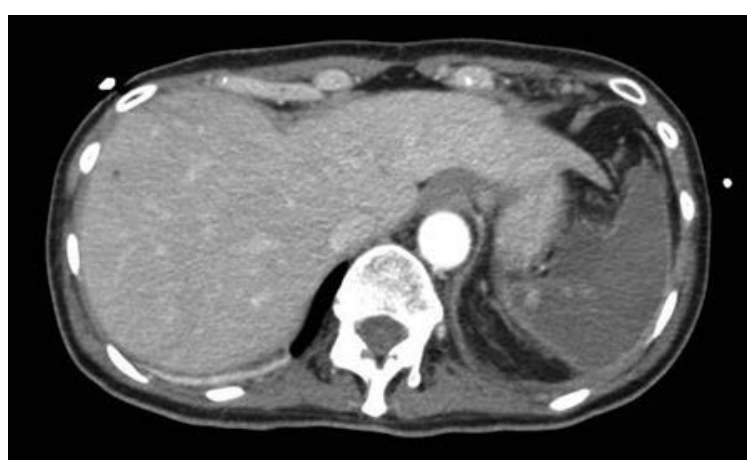

Figure 2. Abdominal computed tomographic scan with contrast medium. mary source of infection, it may be useful to investigate and test for a potential disease causing a functional hyposplenism, sometimes discovered in adulthood as for the patient we described in this report. ${ }^{4}$ In addition, echo-color Doppler with contrast agent is a useful tool for a diagnosis and follow up of patients with SI. This test can be performed on patients' beds and it is particularly useful for non-transportable ICU patients. ${ }^{5}$

\section{References}

1. Schattner A, Adi M, Kitroser E, et al. Acute splenic infarction at an academic general hospital over 10 years. Medicine 2015;94:e1363.

2. Levy M, Toh CH, Thachil J, et al. Guidelines for the diagnosis and management of disseminated intravascular coagulation Br J Haematol 2009;145:24-33.

3. William BM, Corazza GR. Hyposplenism: A comprehensive review. Part I: basic concepts and causes. Hematology 2007;12:1-13.

4. William BM, Thawani N, Sae-Tia S, et al. Hyposplenism: A comprehensive review. Part II: clinical manifestations, diagnosis, and management. Hematology 2007;12:89-98.

5. Ignee A, Atkinson NSS, Schuessler G, et al. Ultrasound contrast agents. Endosc Ultrasound 2016;5:355-62. 